Bueno M, Silva FFF, Gave NC, Costa T, Peres HHC.

[Uso de Tecnologias de Informação e Comunicação no Ensino Sobre Dor: Revisão de Escopo]. Rev Paul Enferm [Internet]. 2019;30. doi:10.33159/25959484 repen.2019v30a5

\section{Uso de Tecnologias de Informação e Comunicação no Ensino Sobre Dor: Revisão de Escopo}

\author{
The Use of Information and Communication Technologies \\ for Teaching about Pain: A Scoping Review \\ Uso de Tecnologías de Información y Comunicación en la \\ Enseñanza sobre Dolor: Revisión de escopo
}

\section{Mariana BuenoI, Fernanda Felipe Ferreira da SilvaII, Nádia Carvalho Gave", Taine Costa ${ }^{\mathrm{IV}}$, Heloisa Helena Ciqueto Peres ${ }^{\mathrm{V}}$}

I Doutora, Pós-doutoranda no The Hospital for Sick Children. Peter Gilgan Centre for Research and Learning (PGCRL) 686 Bay St. Toronto - ON - Canada. M5G 0A4. E-mail: mariana.bueno@sickkids.ca

II Mestre, Enfermeira residente do Programa de Residência Multiprofissional em Atenção Clínica Especializada em Neonatologia do HCFMUSP. End: Av. Dr. Enéas Carvalho de Aguiar, 255 - Cerqueira César, São Paulo - SP, 05403-000. Centro Neonatal 1, 10 Andar Instituto Central. E mail: fernanda.felipe@fm.usp.br

III Enfermeira, supervisão de enfermagem do Pronto Socorro da Santa Casa de Misericórdia de Capivari. End: Praça Doutor Mário Dias de Aguiar, 01, Centro- Capivari- SP, 13330-000. E mail: nadiacgave@gmail.com

IV Mestre, Doutoranda do Programa InterunidadesDoutoramento em Enfermagem da USP, Enfermeira coordenadora assistencial do Hospital Infantil Waldemar Monastier - PR. End: Rua XV de Novembro, 3701 - Bom Jesus - 83601-030 - Campo Largo - PR. E mail: tainecosta@usp.br

V Doutora, Professora Titular do Departamento de Orientação Profissional da EEUSP. End: Av. Dr. Enéas Carvalho de Aguiar, 419 - Cerqueira César, São Paulo - SP, 05403-000. E mail: hhcperes@gmail.com

\section{RESUMO}

Objetivo: Identificar o uso de Tecnologias de Informação e Comunicação (TIC) e o modo como são utilizados no ensino da temática dor a estudantes e profissionais da saúde. Método: Revisão de escopo. A busca foi conduzida em janeiro de 2019 nas bases de dados PubMed, CINAHL, Scopus e BVS. Resultados: 717 referências foram analisadas e 34 estudos foram incluídos. Cursos direcionados a enfermeiros e alunos de enfermagem, com abordagem da dor em indivíduos adultos, e compostos por jogos, vídeos, simulados e imagens foram prevalentes, recebendo avaliações positivas dos usuários. Conclusão: Apesar de pouco descritas na literatura, os resultados indicaram benefícios resultantes do emprego de TIC na educação e capacitação em dor, além da ampla aceitação pelos usuários. O uso de TIC na educação, constitui alternativa inovadora para formação e capacitação profissional na área de saúde, para o ensino da dor e demais especialidades.

Palavras-chave: pessoal de saúde, estudantes de ciência da saúde, tecnologia educacional, tecnologia da informação, dor. 


\section{ABSTRACT}

Objective: To identify the use of Information and Communication Technologies (ICT) to teach about pain and the methods for offering ICTs to students and health professionals. Method: This is a scoping review. The literature search was conducted in January 2019 on PubMed, CINAHL, Scopus and VHL databases. Results: 717 references were reviewed, of which 34 studies were included. There was a prevalence of courses tailored to nurses and nursing students. Courses mainly focused on pain in adults and were comprised of games, videos, simulations, and images. In general, positive feedback was provided by users. Conclusion: The literature describes a limited amount of pain-related ICT. Results from this review indicated benefits related to using ICT in pain education and training, as well as broad acceptance by users. The use of ICT is an innovative alternative for health education and training for pain teaching and other specialties.

Keywords: health personnel, students, health occupations, educational technology, information technology, pain.

\section{RESUMEN}

Objetivo: Identificar el uso de Tecnologías de Información y Comunicación (TIC) y el modo como son utilizados en la enseñanza de la temática dolor a estudiantes y profesionales de la salud. Método: Revisión de escopo conducida en enero de 2019 en las bases de datos PubMed, CINAHL, Scopus y BVS. Resultados: 717 referencias fueron analizadas y 34 estudios fueron incluidos. Cursos direccionados a enfermeros y alumnos de enfermería con abordaje del dolor en individuos adultos y compuestos por juegos, videos, simulados e imágenes fueron prevalentes, recibiendo evaluaciones positivas de los usuarios. Conclusión: A pesar de poco descritas en la literatura, los resultados indicaron beneficios resultantes del empleo de TIC en la educación y capacitación en dolor, además de amplia aceptación por los usuarios. El uso de TC en la educación constituye alternativa innovadora para formación y capacitación profesional en el área de salud para la enseñanza del dolor y demás especialidades. Palabras-clave: personal de salud, estudiantes de ciencias de la salud, tecnología en la educación, tecnología de la información, dolor.

\section{INTRODUÇÃO}

A dor é um fenômeno multidimensional e particular, que acomete o ser humano ao longo de todo ciclo vital. É definida como uma experiência sensorial e emocional desagradável, associada a dano tissular real ou potencial, sendo que cada indivíduo a descreve conforme suas experiências. A ausência de comunicação verbal, contudo, não impede que os indivíduos sintam dor e necessitem de tratamento ${ }^{(1)}$.

Tendo em vista a relevância da dor em todas as fases da vida humana, bem como efeitos potencialmente deletérios, quando não prevenida ou tratada adequadamente, salienta-se a necessidade de inserção deste conteúdo na formação de estudantes e profissionais da área da saúde. Contudo, estudo que objetivou identificar fatores que influenciavam a educação sobre dor, direcionada a alunos de graduação de cursos da saúde, no Reino Unido, evidenciou ausência de disciplina ou cursos específicos sobre o tema, na grade curricular (enfermagem, medicina, obstetrícia, odontologia, farmácia, fisioterapia e terapia ocupacional), além de oferta restrita do conteúdo de modo isolado em diferentes disciplinas. Os alunos avaliaram a grade curricular como limitada, com abordagem inadequada do conteúdo e da carga horária e, ainda, sugeriram o emprego de métodos de ensino inovadores, como o e-learning, estudos de caso e ações de ensino simuladas, com vistas a desenvolver habilidades necessárias para o gerenciamento da dor, o que inclui resolução de problemas, aprendizagem compartilhada, trabalho em equipe e avaliação crítica ${ }^{(2)}$.

Assim, além da necessidade de inclusão do conteúdo relacionado à dor, faz-se necessário, considerar as estratégias educativas empregadas para o ensino de adultos. Para este estudo, destaca-se a utilização de Tecnologias de Informação e Comunicação (TICs). O uso 
de tecnologias pode ser eficaz no aperfeiçoamento do pensamento crítico e de habilidades do indivíduo, com consequente aprimoramento da assistência ${ }^{(3-4)}$.

Observa-se uma vasta gama de terminologias relacionadas às modalidades de tecnologias no âmbito da educação, como educação a distância (EAD), e-learning, uso de dispositivos móveis (m-learning), educação online, educação mediada por computador, entre outros ${ }^{(5)}$. Para este estudo, adotou-se o termo TICs, que engloba a utilização de diferentes modalidades para a disseminação de informações, como Ambiente Virtual de Aprendizagem (AVA), Objetos Virtuais de Aprendizagem (OVA) e softwares ${ }^{(6)}$.

As TICs possibilitam a interação entre professores e alunos separados pelo espaço e/ou tempo, flexibilidade de acesso ao conhecimento e à informação na área de interesse, conforme disponibilidade do usuário, e utilização de diferentes recursos audiovisuais, considerados como ferramentas de apoio ao processo de ensino-aprendizagem ${ }^{(7)}$.

Na educação mediada por TIC, o desenvolvimento de recursos audiovisuais é realizado a partir de elementos do design educacional e princípios pedagógicos ${ }^{(8)}$. Podem ser utilizados em diversos formatos, a exemplo de textos, sons, imagens, animações, vídeos, simulações realísticas, e exercícios de avaliação formativa ${ }^{(9)}$. Os diferentes recursos audiovisuais servem de apoio educacional, favorecendo a interação, a compreensão do conteúdo pelos usuários, 0 desenvolvimento de habilidades e autonomia, e a formação de conceitos e individualidade ${ }^{(10)}$.

Dessa forma, a educação por intermédio das TICS é uma alternativa para formação de estudantes e capacitação de profissionais de saúde em diversas especialidades, inclusive na avaliação e manejo da dor, visto o impacto que a falta de conhecimento traz sobre o cuidado aos pacientes. Assim, o objetivo deste estudo foi identificar o uso das TICs empregadas no ensino da temática dor a estudantes e profissionais de saúde, e o modo como são utilizadas.

\section{MÉTODO}

Trata-se de revisão de escopo, método utilizado para mapear a literatura existente sobre determinado assunto, conduzida em cinco estágios ${ }^{(11-12)}$ : identificação da pergunta de pesquisa; pesquisa de estudos relevantes; seleção de estudos; mapeamento e comparação dos dados; e, síntese e apresentação de resultados. Adicionalmente, utilizou-se a estratégia Preferred Reporting Items for Systematic Reviews and Meta-Analyses (PRISMA)(13) para descrição desta revisão.

No primeiro estágio, identificação da pergunta de pesquisa, foram identificadas as fragilidades da literatura sobre o tema abordado, pois não foram encontradas revisões de literatura relacionadas a esta temática, por isso, a seguinte pergunta norteou o estudo: Quais e de que forma as TICs são utilizadas para o ensino da dor à estudantes e aos profissionais da área da saúde?

No segundo estágio, pesquisa de estudos relevantes, foram incluídos estudos que apresentassem dados primários, sem delimitação de desenho, ano de publicação e idioma, e que tiveram como objeto, a investigação de estratégias de educação sobre dor, à distância e mediadas por tecnologia, direcionadas a estudantes e profissionais da saúde. No terceiro estágio, seleção dos estudos, foram excluídas as pesquisas que descreveram estratégias de educação presenciais, com ou sem uso de TIC, estudos cujas estratégias de ensino não desenvolveram a temática dor, estudos cujas estratégias educacionais eram direcionadas a pacientes ou familiares, resumos publicados em anais de congressos, manuais, livros, comunicações, comentários, cartas ou editoriais e, por fim, os estudos cujos textos completos não puderam ser obtidos, mesmo após extensa busca ou solicitação aos autores.

Neste estágio, ainda, adotou-se a estratégia PICO adaptada (14), PICo (P: população, I: intervenção, Co: contexto) guiou a elaboração da pergunta norteadora desta revisão de escopo e serviu de base para o desenvolvimento das estratégias de busca. Para população, os seguintes termos 
foram empregados nas buscas: health care professionals, healthcare professionals, health personnel, health care providers, health care team, health providers team, healthcare providers, healthcare team, healthcare personnel, health occupation students. Quanto à intervenção, os termos usados foram: distance education, distance learning, e-learning, online training, web-based learning, correspondence courses, online capacitation, educational technology, internet-based training, online learning, internet learning. Finalmente, para contexto, utilizaram-se os seguintes termos: pain, pain assessment, pain measurement, pain management. As combinações foram realizadas respeitando-se as particularidades de cada base de dados e utilizando-se operadores booleanos OR e AND.

A busca foi realizada em janeiro de 2019 na biblioteca virtual BVS e nas bases de dados eletrônicas CINAHL, Scopus e MEDLINE (via Pubmed). Para busca na literatura cinzenta foram utilizadas, a Biblioteca Digital de Teses e Dissertações da Universidade de São Paulo, e recursos de redes sociais acadêmicas como Google Acadêmico, Academia.edu e Research Gate. Além disso, foram realizadas buscas adicionais a partir das referências dos próprios artigos incluídos no presente estudo.

No estágio de mapeamento e comparação dos dados, quarto estágio, os títulos e resumos recuperados foram avaliados por duas revisoras de modo independente (FS e NG ou FS e TC) e as divergências foram resolvidas por um terceiro revisor (TC ou MB). Posteriormente, as referências consideradas pertinentes foram acessadas na íntegra para análise pelas mesmas revisoras, ainda de forma independente. Para síntese e apresentação dos artigos selecionados, foram analisados os seguintes itens de cada artigo: título, autores, ano, país de publicação, público-alvo, objetivos do estudo e da estratégia educacional, desenvolvimento da estratégia educacional, utilização de recursos educacionais, formas de avaliação e principais resultados. Para finalizar, o quinto estágio, composto de síntese e apresentação dos dados, é apresentado a seguir, de forma descritiva.

\section{RESULTADOS}

Foram recuperados 717 referências, das quais 359 no PubMed, 147 no SCOPUS, 136 no CINAHL e 75 encontrados na BVS. Foram incluídos nesta revisão 34 estudos, dos quais 27 foram identificados a partir das bases de dados e sete artigos, com base na análise das referências de estudos incluídos, conforme Figura 1.

Há prevalência de estudos norte-americanos (19-55,8\%), seguidos por estudos do Reino Unido ( $3-8,8 \%$ ) e estudos brasileiros (3-8,8\%). A maioria das estratégias educativas envolvendo TICs foi direcionada exclusivamente a enfermeiros e alunos de graduação em enfermagem ( $15-44,1 \%)$ e a médicos, residentes e alunos de graduação em medicina (7 - 20,5\%). A caracterização dos estudos incluídos está descrita nos Quadros 1 e 2, a seguir.

Conforme os dados descritos nos Quadros 1 e 2, foram encontrados 16 (47\%) estudos quasi-experimentais, sete (20,5\%) ensaios clínicos randomizados, seis (17,6\%) estudos transversais, dois $(5,8 \%)$ relatos de caso, dois $(5,8 \%)$ coortes e um $(2,94 \%)$ método misto. Quanto à faixa etária, 27 (79,4\%) estudos referiam-se à TIC sobre dor em adultos, quatro $(11,7 \%)$ à dor pediátrica, dois $(5,8 \%)$ à dor neonatal e um $(5,8 \%)$ abordou tanto a dor neonatal quanto em adultos.

As TICs identificadas nesta revisão incluíram software, blog, email, AVA, OVA e CD-ROM. Os recursos audiovisuais empregados foram animações, exercícios interativos, gráficos, questões de múltipla escolha, textos, quizzes, imagens, vídeos, estudos de caso, auditoria, feedback, seção de ajuda aos usuários, áudios, links para websites, pacientes virtuais, tutoriais, elementos de videogames, exercícios, textos complementares, fórum de discussão, slides, palavras cruzadas, vídeo-aulas e podcasts.

Nos estudos analisados, os usuários apontaram aspectos positivos do conteúdo e aparência das TICs, como: navegabilidade, clareza de informações, pertinência dos objetivos e conteúdo propostos, estética do layout, recursos audiovisuais, referências utilizadas, interatividade, carga horária, feedback, tutores e ajuda ao usuário. 


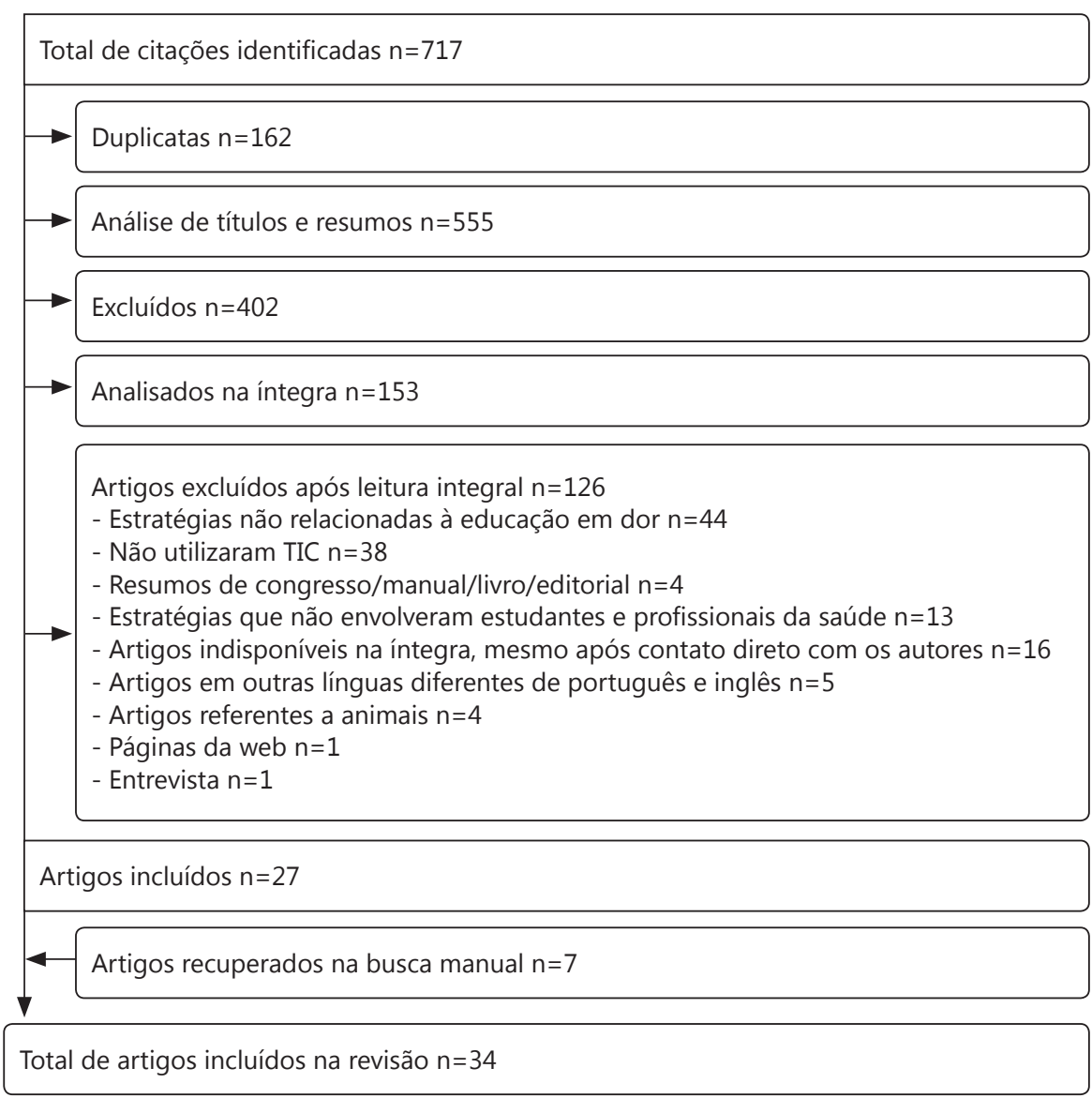

Figura 1

Quadro 1 - Síntese dos estudos incluídos que tratam de estratégias educativas sobre dor em adultos, segundo primeiro autor, país e ano de publicação, público-alvo, desenho do estudo, TIC, recursos audiovisuais, avaliação e principais resultados - São Paulo, SP, 2019.

\begin{tabular}{|c|c|c|c|c|}
\hline $\begin{array}{c}\text { Primeiro } \\
\text { autor/ País/ } \\
\text { Ano }\end{array}$ & $\begin{array}{l}\text { Público-alvo } \\
\text { (População/amostra) } \\
\text { Desenho do estudo }\end{array}$ & $\begin{array}{c}\text { TIC } \\
\text { Recursos } \\
\text { Audiovisuais }\end{array}$ & Avaliação & Principais Resultados \\
\hline $\begin{array}{l}\text { Huckstadt }{ }^{(15)} \\
\text { EUA } \\
2005\end{array}$ & $\begin{array}{c}\text { Enfermeiros (73) } \\
\text { Quasi-experimental }\end{array}$ & $\begin{array}{l}\text { AVA } \\
\text { Estudos de caso, } \\
\text { feedback, seção } \\
\text { de ajuda ao } \\
\text { usuário e estudos } \\
\text { de caso }\end{array}$ & $\begin{array}{c}\text { Pré-teste, pós-teste } \\
\text { e avaliação do } \\
\text { curso }\end{array}$ & $\begin{array}{l}\text { A média de acertos no pós-teste foi maior em } \\
\text { relação ao pré-teste. Os usuários mostraram-se } \\
\text { satisfeitos com o caráter inovador e flexível do } \\
\text { curso, e os gráficos e conteúdos dos estudos de } \\
\text { caso. Os usuários relataram que aprenderam mais } \\
\text { com as TICs do que com ensino tradicional. }\end{array}$ \\
\hline $\begin{array}{l}\text { Dy } \\
\text { EUA } \\
2008\end{array}$ & $\begin{array}{l}\text { Residentes de medicina } \\
\qquad(612) \\
\text { Quasi-experimental }\end{array}$ & $\begin{array}{l}\text { AVA } \\
\text { Estudos de } \\
\text { caso, websites } \\
\text { e questões de } \\
\text { múltipla-escolha. }\end{array}$ & $\begin{array}{c}\text { Pré-teste, pós-teste } \\
\text { e avaliação do } \\
\text { curso }\end{array}$ & $\begin{array}{l}\text { A média de acertos do pós-teste foi maior em } \\
\text { relação ao pré-teste. Os usuários apontaram que o } \\
\text { curso contribuirá para melhorar a assistência dos } \\
\text { pacientes. }\end{array}$ \\
\hline $\begin{array}{l}\text { Yanni }^{(17)} \\
\text { EUA } \\
2009\end{array}$ & $\begin{array}{l}\text { Médicos, residentes e } \\
\text { alunos de graduação em } \\
\text { medicina (439) } \\
\text { Quasi-experimental }\end{array}$ & $\begin{array}{l}\text { AVA } \\
\text { Estudos de } \\
\text { caso, leitura } \\
\text { complementar } \\
\text { e links para } \\
\text { websites úteis. }\end{array}$ & $\begin{array}{c}\text { Pré-teste, pós-teste } \\
\text { e avaliação do } \\
\text { curso. }\end{array}$ & $\begin{array}{l}\text { Não houve diferença significativa entre os } \\
\text { resultados do pré-teste e pós-teste. Os alunos } \\
\text { e residentes acharam o curso difícil, porém os } \\
\text { médicos consideraram o conteúdo pertinente e de } \\
\text { fácil compreensão. }\end{array}$ \\
\hline
\end{tabular}


Continuação do Quadro 1

\begin{tabular}{|c|c|c|c|c|}
\hline $\begin{array}{l}\text { Primeiro } \\
\text { autor/ País/ } \\
\text { Ano }\end{array}$ & $\begin{array}{l}\text { Público-alvo } \\
\text { (População/amostra) } \\
\text { Desenho do estudo }\end{array}$ & $\begin{array}{c}\text { TIC } \\
\text { Recursos } \\
\text { Audiovisuais }\end{array}$ & Avaliação & Principais Resultados \\
\hline $\begin{array}{l}\text { Alvarez }^{(18)} \\
\text { Brasil } \\
2011\end{array}$ & $\begin{array}{l}\text { Estudantes de enfermagem } \\
\qquad(14) \\
\text { Quasi-experimental }\end{array}$ & $\begin{array}{c}\text { AVA } \\
\text { Slides, cruzadas, } \\
\text { leitura } \\
\text { complementar, } \\
\text { links para sites } \\
\text { úteis, pacientes } \\
\text { virtuais e } \\
\text { instrumentos de } \\
\text { avaliação da dor. }\end{array}$ & $\begin{array}{c}\text { Pré-teste, pós-teste } \\
\text { e avaliação do } \\
\text { curso. }\end{array}$ & $\begin{array}{l}\text { A média de acertos no pós-teste foi maior } \\
\text { em relação ao pré-teste. A flexibilidade e a } \\
\text { aprendizagem, auto direcionadas, foram vantagens } \\
\text { apontadas. Como limitações, destacaram-se, } \\
\text { dificuldades técnicas e de interpretação dos } \\
\text { indicadores de dor nos pacientes virtuais. }\end{array}$ \\
\hline $\begin{array}{l}\text { Puljak }{ }^{(19)} \\
\text { Croácia } \\
2011\end{array}$ & $\begin{array}{c}\text { Estudantes de medicina e } \\
\text { odontologia (73) } \\
\text { Quasi-experimental }\end{array}$ & $\begin{array}{l}\text { AVA } \\
\text { Tutorias e fóruns } \\
\text { de discussão }\end{array}$ & $\begin{array}{l}\text { Pré-teste, pós-teste } \\
\text { e avaliação do } \\
\text { curso. }\end{array}$ & $\begin{array}{l}\text { Houve aumento nos acertos do pós-teste em } \\
\text { relação ao pré-teste. Os usuários indicaram } \\
\text { vantagens no uso de TICs, como: facilidade para } \\
\text { estudar, buscar informações e melhor comunicação } \\
\text { com os professores e seus pares. }\end{array}$ \\
\hline $\begin{array}{l}\text { Borglin }^{(20)} \\
\text { Suécia } 2014\end{array}$ & $\begin{array}{l}\text { Enfermeiros (34) } \\
\text { Quasi-experimental }\end{array}$ & $\begin{array}{c}\text { AVA } \\
\text { Não informado }\end{array}$ & $\begin{array}{l}\text { Avaliação de } \\
\text { prontuário }\end{array}$ & $\begin{array}{l}\text { Houve redução nos registros de dor referida pelos } \\
\text { pacientes após a intervenção. }\end{array}$ \\
\hline $\begin{array}{l}\text { Phillips(21) } \\
\text { Austrália, } \\
2014\end{array}$ & $\begin{array}{c}\text { Enfermeiros (74) } \\
\text { Quasi-experimental }\end{array}$ & $\begin{array}{l}\text { software } \\
\text { Estudos de caso }\end{array}$ & $\begin{array}{l}\text { Pré-teste, pós- } \\
\text { teste, avaliação } \\
\text { de prontuário e } \\
\text { avaliação do curso }\end{array}$ & $\begin{array}{l}\text { Não houve diferença significativa entre os } \\
\text { resultados do pré e do pós-teste, nem diminuição } \\
\text { do escore de dor dos pacientes. }\end{array}$ \\
\hline $\begin{array}{l}\text { Slaughter(22) } \\
\text { EUA } \\
2015\end{array}$ & $\begin{array}{l}\text { Enfermeiros (80) } \\
\text { Quasi-experimental }\end{array}$ & $\begin{array}{l}\text { AVA } \\
\text { Textos, links para } \\
\text { websites úteis, } \\
\text { vídeos e tutoriais. }\end{array}$ & $\begin{array}{l}\text { Pré-teste, pós-teste } \\
\text { e avaliação do } \\
\text { curso. }\end{array}$ & $\begin{array}{l}\text { A média de acertos no pós-teste, tanto na área de } \\
\text { conhecimentos, quanto em relação à percepção } \\
\text { do profissional sobre dor, foi mais alta, em relação } \\
\text { ao pré-teste. Os enfermeiros avaliaram o curso de } \\
\text { forma positiva. }\end{array}$ \\
\hline $\begin{array}{l}\text { Friesgaard (23) } \\
\text { Dinamarca } \\
2017\end{array}$ & $\begin{array}{l}\text { Enfermeiros (53) } \\
\text { Residentes de medicina } \\
\qquad(14) \\
\text { Quasi-experimental }\end{array}$ & $\begin{array}{l}\text { AVA } \\
\text { Estudos caso } \\
\text { interativo }\end{array}$ & Pré-teste, pós-teste & $\begin{array}{l}\text { O conhecimento sobre manejo da dor aumentou } \\
\text { em relação ao pré-teste, porém, como limitação do } \\
\text { estudo, o autor destaca que não foi observada a } \\
\text { aplicação do conhecimento na prática clínica. }\end{array}$ \\
\hline $\begin{array}{l}\text { Phillips (24) } \\
\text { Australia } \\
2017\end{array}$ & $\begin{array}{l}\text { Enfermeiros (44) } \\
\text { Quasi-experimental }\end{array}$ & $\begin{array}{l}\text { AVA } \\
\text { Estudos de } \\
\text { caso, auditoria e } \\
\text { feedback }\end{array}$ & Pré-teste, pós-teste & $\begin{array}{l}\text { O conhecimento sobre avaliação da dor, } \\
\text { instrumento de avaliação, e a confiança na } \\
\text { avaliação, aumentaram em relação ao pré-teste. } \\
\text { Houve aumento nos registros de avaliações de dor, } \\
\text { documentadas nos prontuários. }\end{array}$ \\
\hline $\begin{array}{l}\text { Bonkowski }{ }^{(25)} \\
\text { EUA } \\
2018\end{array}$ & $\begin{array}{l}\text { Enfermeiros (26) } \\
\text { Quasi-experimental }\end{array}$ & $\begin{array}{c}\text { AVA } \\
\text { Não informado }\end{array}$ & Pré-teste, pós-teste & $\begin{array}{l}\text { Não houve mudanças significativas no pré e pós } \\
\text { teste sobre atitudes dos enfermeiros frente à dor, } \\
\text { havendo mudanças na prática assistencial. }\end{array}$ \\
\hline $\begin{array}{l}\text { Watt- } \\
\text { Watson(26) } \\
\text { Canadá } \\
2018\end{array}$ & $\begin{array}{c}\text { Profissionais de saúde (96) } \\
\text { Quasi-experimental }\end{array}$ & $\begin{array}{l}\text { AVA } \\
\text { Conteúdo escrito, } \\
\text { roteiros em vídeo } \\
\text { e exercícios de } \\
\text { reflexão }\end{array}$ & $\begin{array}{l}\text { Pré-teste, pós- } \\
\text { teste, escala de } \\
\text { Likert }\end{array}$ & $\begin{array}{l}\text { Os escores gerais de usabilidade foram elevados. } \\
\text { Em média, os escores de conhecimento sobre dor } \\
\text { aumentaram } 20 \% \text { e houve melhora significativa } \\
\text { sobre a compreensão do cuidado colaborativo. }\end{array}$ \\
\hline $\begin{array}{l}\text { Johnson }^{(27)} \\
\text { EUA } \\
1997\end{array}$ & $\begin{array}{l}\text { Estudantes de enfermagem } \\
\qquad \begin{array}{c}\text { (19) } \\
\text { ECR } \\
\text { GC = aula presencial } \\
\text { X } \\
\text { GI = TIC }\end{array}\end{array}$ & $\begin{array}{l}\text { software } \\
\text { Animações, } \\
\text { exercícios } \\
\text { interativos e } \\
\text { gráficos }\end{array}$ & $\begin{array}{l}\text { Pré-teste, pós-teste } \\
\text { e avaliação do } \\
\text { curso }\end{array}$ & $\begin{array}{l}\text { Em relação aos aspectos clínicos da dor, ambos } \\
\text { os grupos obtiveram maior pontuação no pós- } \\
\text { teste em comparação ao pré-teste. Os alunos } \\
\text { do GI relataram que o curso permitiu o controle } \\
\text { de seu aprendizado, respeitando-se o ritmo e as } \\
\text { dificuldades. }\end{array}$ \\
\hline $\begin{array}{l}\text { Raffety } \\
\text { EUA } \\
2000\end{array}$ & $\begin{array}{c}\text { Enfermeiros, médicos e } \\
\text { estudantes de enfermagem } \\
\text { e medicina (40) } \\
\text { ECR } \\
\text { GC = aula presencial } \\
\text { X } \\
\text { GI = TIC }\end{array}$ & $\begin{array}{l}\text { AVA } \\
\text { Questões de } \\
\text { múltipla-escolha }\end{array}$ & $\begin{array}{l}\text { Pré-teste, pós-teste } \\
\text { e avaliação do } \\
\text { curso }\end{array}$ & $\begin{array}{l}\text { O GI obteve média de acertos no pós-teste três } \\
\text { vezes maior do que o GC. Os usuários do GI } \\
\text { avaliaram positivamente a estratégia. }\end{array}$ \\
\hline
\end{tabular}


Continuação do Quadro 1

\begin{tabular}{|c|c|c|c|c|}
\hline $\begin{array}{l}\text { Primeiro } \\
\text { autor/ País/ } \\
\text { Ano }\end{array}$ & $\begin{array}{l}\text { Público-alvo } \\
\text { (População/amostra) } \\
\text { Desenho do estudo }\end{array}$ & $\begin{array}{l}\text { TIC } \\
\text { Recursos } \\
\text { Audiovisuais }\end{array}$ & Avaliação & Principais Resultados \\
\hline $\begin{array}{l}\text { Harris }^{(29)} \\
\text { EUA } \\
2008\end{array}$ & $\begin{array}{l}\text { Médicos (95) } \\
\text { ECR } \\
\text { GC = palestras } \\
\text { X } \\
\text { GI = TIC }\end{array}$ & $\begin{array}{l}\text { AVA } \\
\text { Pacientes virtuais, } \\
\text { tutoriais, vídeos, } \\
\text { elementos de } \\
\text { videogames e } \\
\text { exercícios. }\end{array}$ & $\begin{array}{l}\text { Pré-teste, pós-teste } \\
\text { imediato, pós-teste } \\
\text { de follow-up e } \\
\text { avaliação do curso }\end{array}$ & $\begin{array}{l}\text { Houve aumento significativo nos resultados do pós- } \\
\text { teste imediato e de follow-up do GI, e mostraram- } \\
\text { se satisfeitos com a intervenção. }\end{array}$ \\
\hline $\begin{array}{l}\text { Brody }^{(30)} \\
\text { EUA } \\
2013\end{array}$ & $\begin{array}{c}\text { Enfermeiros (10) } \\
\text { ECR } \\
\text { GC = aulas presenciais } \\
X \\
\text { GI }=\text { TIC }\end{array}$ & $\begin{array}{c}\text { AVA } \\
\text { Textos, áudios, } \\
\text { estudos de } \\
\text { caso e questões } \\
\text { múltipla-escolha. }\end{array}$ & $\begin{array}{l}\text { Pré e pós-teste } \\
\text { imediato e follow- } \\
\text { up e avaliação do } \\
\text { curso }\end{array}$ & $\begin{array}{l}\text { Houve aumento significativo no pós-teste imediato } \\
\text { do GI, porém não se notou diferença no follow- } \\
\text { up. Os usuários avaliaram a intervenção como } \\
\text { útil e pertinente e sugeriram incluir mais recursos } \\
\text { audiovisuais. }\end{array}$ \\
\hline $\begin{array}{l}\text { Weiner }{ }^{(31)} \\
\text { EUA } \\
2014\end{array}$ & $\begin{array}{l}\text { Estudantes de medicina (55) } \\
\qquad \begin{array}{c}\text { ECR } \\
\text { GC = sem intervenção } \\
\text { X } \\
\text { GI = TIC }\end{array}\end{array}$ & $\begin{array}{c}\text { AVA } \\
\text { Estudos de caso } \\
\text { com pacientes } \\
\text { virtuais, vídeos e } \\
\text { slides. }\end{array}$ & $\begin{array}{c}\text { Pré-teste e pós- } \\
\text { teste }\end{array}$ & $\begin{array}{l}\text { O GI apresentou melhor desempenho na estação } \\
\text { prática de dor geriátrica, após a intervenção, em } \\
\text { comparação com o GC. }\end{array}$ \\
\hline $\begin{array}{l}\text { Trudeau }{ }^{(32)} \\
\text { EUA } \\
2017\end{array}$ & $\begin{array}{c}\text { Médicos, residentes e } \\
\text { enfermeiros (238) } \\
\text { ECR } \\
\text { GC = curso online já } \\
\text { existente } \\
\text { X } \\
\text { GI = TIC (nova modalidade } \\
\text { de curso online) }\end{array}$ & $\begin{array}{c}\text { AVA } \\
\text { Estudos de caso } \\
\text { com pacientes } \\
\text { virtuais, } \\
\text { vídeos, leitura } \\
\text { complementar }\end{array}$ & $\begin{array}{l}\text { Pré-teste, pós- } \\
\text { teste, formulário } \\
\text { específico sobre } \\
\text { dor em adultos } \\
\text { que avalia } \\
\text { comportamentos e } \\
\text { atitudes, satisfação } \\
\text { com o curso }\end{array}$ & $\begin{array}{l}\text { Tanto os participantes do GC como os do GI } \\
\text { aumentaram seus comportamentos sobre a prática } \\
\text { de dor, reduziram suas atitudes negativas e suas } \\
\text { relutâncias e desconfortos para utilizarem opióides } \\
\text { com segurança para os pacientes. }\end{array}$ \\
\hline $\begin{array}{l}\text { Jacobs }{ }^{(33)} \\
\text { EUA } \\
2018\end{array}$ & $\begin{array}{c}\text { Residentes de medicina } \\
\qquad \begin{array}{c}(143) \\
\text { ECR } \\
\text { GC = literatura } \\
X \\
\text { GI = TIC }\end{array}\end{array}$ & $\begin{array}{c}\text { AVA } \\
\text { Videoclipe }\end{array}$ & Pré-teste, pós-teste & $\begin{array}{l}\text { Escores de conhecimento permaneceram os } \\
\text { mesmos em ambos os grupos, com tendências } \\
\text { para melhorias no grupo de intervenção. O GI } \\
\text { apresentou melhor qualidade na realização do } \\
\text { exame físico. }\end{array}$ \\
\hline $\begin{array}{l}\text { Sloan }^{(34)} \\
\text { EUA } \\
2002\end{array}$ & $\begin{array}{c}\text { Estudantes de medicina } \\
\qquad(11) \\
\text { Transversal }\end{array}$ & $\begin{array}{l}\text { CD-ROM } \\
\text { Instruções } \\
\text { e vídeos }\end{array}$ & $\begin{array}{l}\text { Auto avaliação e } \\
\text { avaliação do curso }\end{array}$ & $\begin{array}{l}\text { Os usuários apontaram que o curso foi bem } \\
\text { organizado, fácil de usar e aprimorou o } \\
\text { conhecimento. A maioria dos participantes } \\
\text { considerou que os vídeos utilizados, contudo, foram } \\
\text { maçantes. }\end{array}$ \\
\hline $\begin{array}{l}\text { Douglass }^{(35)} \\
\text { EUA } \\
2015\end{array}$ & $\begin{array}{l}\text { Estudantes de farmácia } \\
\qquad(164) \\
\text { Transversal }\end{array}$ & $\begin{array}{c}\text { AVA } \\
\text { Não informado }\end{array}$ & Auto avaliação & $\begin{array}{l}\text { Todos os alunos avaliaram-se pouco confiantes } \\
\text { no manejo da dor crônica. Todos reportaram } \\
\text { muita confiança no manejo da dor aguda, porém, } \\
\text { demonstraram pouca competência nessa área. }\end{array}$ \\
\hline $\begin{array}{l}\text { Fricton }^{(36)} \\
\text { EUA } \\
2015\end{array}$ & $\begin{array}{l}\text { Profissionais e estudantes } \\
\text { da área da saúde e } \\
\text { pacientes (771) } \\
\text { Transversal }\end{array}$ & $\begin{array}{l}\text { AVA } \\
\text { Palestras por } \\
\text { vídeo, exercícios } \\
\text { e fóruns de } \\
\text { discussão. }\end{array}$ & Avaliação do curso. & $\begin{array}{l}\text { A maioria dos usuários avaliou positivamente } \\
\text { o curso, e relatou que o mesmo foi relevante e } \\
\text { aplicável na prática clínica. Os usuários indicaram } \\
\text { que os vídeos e os fóruns de discussão foram } \\
\text { excelentes recursos. }\end{array}$ \\
\hline $\begin{array}{c}\text { Terpstraa } \\
\text { Holanda } \\
2018\end{array}$ & $\begin{array}{c}\text { Profissionais de saúde } \\
\text { mental (22) } \\
\text { Transversal }\end{array}$ & $\begin{array}{l}\text { AVA } \\
\text { Estudos de caso, } \\
\text { simulados }\end{array}$ & $\begin{array}{c}\text { Questionário } \\
\text { de satisfação e } \\
\text { avaliação do curso }\end{array}$ & $\begin{array}{l}\text { Após o treinamento, os profissionais obtiveram } \\
\text { atitudes positivas quanto à assistência ao paciente } \\
\text { com dor. Quanto a satisfação, os participantes } \\
\text { avaliaram o treinamento online como útil e fácil de } \\
\text { usar. }\end{array}$ \\
\hline $\begin{array}{c}\text { Keefe }^{(38)} \\
\text { Reino Unido } \\
2012\end{array}$ & $\begin{array}{l}\text { Estudantes de enfermagem } \\
\qquad \begin{array}{c}\text { (206) } \\
\text { Coorte } \\
\text { GC = aulas presenciais } \\
\text { X } \\
\text { GI = TIC }\end{array}\end{array}$ & $\begin{array}{l}\text { OVA } \\
\text { Imagens, quizzes, } \\
\text { exercícios, } \\
\text { áudios, textos e } \\
\text { links de websites. }\end{array}$ & $\begin{array}{l}\text { Exercícios de } \\
\text { avaliação }\end{array}$ & $\begin{array}{l}\text { Os grupos que utilizaram o recurso tecnológico, } \\
\text { obtiveram maior pontuação nos dois módulos em } \\
\text { relação aos grupos que participaram apenas de } \\
\text { aulas presenciais. }\end{array}$ \\
\hline
\end{tabular}




\begin{tabular}{|c|c|c|c|c|}
\hline $\begin{array}{l}\text { Primeiro } \\
\text { autor/ País/ } \\
\text { Ano }\end{array}$ & $\begin{array}{l}\text { Público-alvo } \\
\text { (População/amostra) } \\
\text { Desenho do estudo }\end{array}$ & $\begin{array}{c}\text { TIC } \\
\text { Recursos } \\
\text { Audiovisuais }\end{array}$ & Avaliação & Principais Resultados \\
\hline $\begin{array}{l}\text { Jenkins }{ }^{(39)} \\
\text { Reino Unido } \\
2014\end{array}$ & $\begin{array}{c}\text { Médicos, enfermeiros, } \\
\text { farmacêuticos e } \\
\text { fisioterapeutas (48) } \\
\text { Coorte } \\
\text { (4 coortes de acordo com a } \\
\text { profissão) }\end{array}$ & $\begin{array}{l}\text { AVA e blog } \\
\text { Vídeo-aulas, aulas } \\
\text { em PowerPoint, } \\
\text { podcasts, } \\
\text { textos e leitura } \\
\text { complementar. }\end{array}$ & $\begin{array}{l}\text { Exercício de } \\
\text { avaliação }\end{array}$ & $\begin{array}{l}\text { A maioria dos usuários mostrou-se satisfeita } \\
\text { com o curso e os conteúdos abordados. Alguns } \\
\text { participantes referiram que o curso foi decisivo } \\
\text { para mudar e aprimorar a prática clínica e suas } \\
\text { habilidades. }\end{array}$ \\
\hline $\begin{array}{l}\text { Schmitt(40) } \\
\text { EUA } \\
2004\end{array}$ & $\begin{array}{c}\text { Enfermeiros (129) } \\
\text { Relato de Caso }\end{array}$ & $\begin{array}{l}\text { AVA } \\
\text { Textos, } \\
\text { quizzes e } \\
\text { imagens. }\end{array}$ & Avaliação do curso & $\begin{array}{l}\text { A avaliação foi positiva. Sugestões incluíram facilitar } \\
\text { a navegabilidade, resumir informações e utilizar } \\
\text { mais recursos audiovisuais. A falta de tempo e de } \\
\text { habilidade no uso de tecnologias foram dificuldades } \\
\text { apontadas. }\end{array}$ \\
\hline $\begin{array}{l}\text { Jansen }^{(41)} \\
\text { Irlanda } \\
2018\end{array}$ & $\begin{array}{l}\text { Enfermeiros (20) } \\
\text { médicos (17) } \\
\text { assistentes de saúde (1) } \\
\text { Método Misto }\end{array}$ & Teleconferência & $\begin{array}{l}\text { Pré-teste, pós- } \\
\text { teste, grupos focais }\end{array}$ & $\begin{array}{l}\text { Houve melhora no conhecimento dos profissionais } \\
\text { envolvidos no estudo, em relação ao pré-teste. } \\
\text { Pelos grupos focais, os participantes consideraram } \\
\text { ganho significativo de conhecimento e habilidades } \\
\text { novas, bem como reconheceram a importância de } \\
\text { aperfeiçoamento para o manejo da dor. }\end{array}$ \\
\hline
\end{tabular}

GC: Grupo controle; GI: Grupo intervenção; ECR: ensaio clínico randomizado.

Quadro 2 - Síntese dos estudos incluídos que tratam de estratégias educativas sobre dor em pediatria e neonatologia segundo primeiro autor, país e ano de publicação, público-alvo, desenho do estudo, TIC, recursos audiovisuais, avaliação e principais resultados - São Paulo, SP, 2019.

\begin{tabular}{|c|c|c|c|c|}
\hline $\begin{array}{l}\text { Primeiro } \\
\text { autor/ País/ } \\
\text { Ano }\end{array}$ & $\begin{array}{c}\text { Público-alvo } \\
\text { (População/amostra) } \\
\text { Desenho do estudo }\end{array}$ & $\begin{array}{c}\text { TIC } \\
\text { Recursos } \\
\text { Audiovisuais }\end{array}$ & Avaliação & Principais Resultados \\
\hline $\begin{array}{l}\text { Vincent }^{(42)} \\
\text { EUA } \\
2011\end{array}$ & $\begin{array}{c}\text { Enfermeiros (24) } \\
\text { Quasi-experimental }\end{array}$ & $\begin{array}{l}\text { AVA } \\
\text { Imagens, vídeos, } \\
\text { áudios, gráficos. }\end{array}$ & $\begin{array}{l}\text { Pré-teste, pós-teste } \\
\text { e avaliação de } \\
\text { prontuário do } \\
\text { paciente. }\end{array}$ & $\begin{array}{l}\text { Dor pediátrica } \\
\text { A maioria dos usuários relatou que o curso é fácil de } \\
\text { entender e utilizar. Houve aumento nos resultados } \\
\text { do pós-teste em relação ao pré-teste. Notou-se } \\
\text { diminuição no escore de dor das crianças e melhora } \\
\text { na administração de analgésicos. }\end{array}$ \\
\hline $\begin{array}{l}\text { Ameringer } \\
\text { (43) } \\
\text { EUA } \\
2012\end{array}$ & $\begin{array}{l}\text { Estudantes de medicina } \\
\qquad(291) \\
\text { Quasi-experimental }\end{array}$ & $\begin{array}{l}\text { AVA } \\
\text { Links, referências, } \\
\text { estudos de caso e } \\
\text { exercícios. }\end{array}$ & $\begin{array}{c}\text { Pré-teste, pós-teste } \\
\text { e avaliação do } \\
\text { curso. }\end{array}$ & $\begin{array}{l}\text { Dor pediátrica } \\
\text { Houve aumento significativo nos acertos do } \\
\text { pós-teste em relação ao pré-teste. A maioria dos } \\
\text { usuários avaliou positivamente o módulo e referiu } \\
\text { que o curso é de fácil utilização. }\end{array}$ \\
\hline $\begin{array}{l}\text { Habich }^{(44)} \\
\text { EUA } \\
2015\end{array}$ & $\begin{array}{c}\text { Enfermeiros (78) } \\
\text { Quasi-experimental }\end{array}$ & $\begin{array}{c}\text { software } \\
\text { Apresentação } \\
\text { narrada do curso. }\end{array}$ & $\begin{array}{l}\text { Pré-teste, pós- } \\
\text { teste, avaliação } \\
\text { de prontuário e } \\
\text { avaliação do curso. }\end{array}$ & $\begin{array}{l}\text { Dor pediátrica } \\
\text { Houve aumento significativo entre os acertos do } \\
\text { pós-teste. A maioria dos participantes acredita que } \\
\text { o curso atingiu os objetivos e que o conteúdo é } \\
\text { pertinente. Os usuários realizaram adequadamente } \\
\text { registro de avaliação e manejo da dor. }\end{array}$ \\
\hline $\begin{array}{l}{ }^{*} \text { Alvarez } \\
\text { Brasil }^{(45)} \\
2017\end{array}$ & $\begin{array}{c}\text { Estudantes de } \\
\text { enfermagem (75) } \\
\text { Quasi-experimental }\end{array}$ & $\begin{array}{l}\text { OVA } \\
\text { Exercícios, links de } \\
\text { websites úteis e } \\
\text { feedback. }\end{array}$ & $\begin{array}{l}\text { Pré-teste, pós-teste } \\
\text { e avaliação do } \\
\text { curso. }\end{array}$ & $\begin{array}{l}\text { Dor neonatal e em adultos } \\
\text { A maioria dos estudantes obteve pontuação } \\
\text { maior no pós-teste em relação ao pré-teste. Em } \\
\text { relação a satisfação dos usuários, a maioria indicou } \\
\text { que a estratégia foi enriquecedora, e avaliaram } \\
\text { positivamente o uso da tecnologia persuasiva no } \\
\text { processo de aprendizagem. }\end{array}$ \\
\hline $\begin{array}{l}\text { Gibbins(46) } \\
\text { Canadá } \\
2007\end{array}$ & $\begin{array}{l}\text { Enfermeiros, médicos, } \\
\text { fisioterapeutas, terapeutas } \\
\text { ocupacionais (68) } \\
\text { Transversal }\end{array}$ & $\begin{array}{l}\text { AVA } \\
\text { Vídeos, imagens, } \\
\text { áudios, questões } \\
\text { de múltipla- } \\
\text { escolha e estudos } \\
\text { de caso. }\end{array}$ & Avaliação do curso & $\begin{array}{l}\text { Dor neonatal } \\
\text { Os usuários avaliaram positivamente o formato } \\
\text { modular do curso, as instruções, a linguagem e os } \\
\text { recursos. }\end{array}$ \\
\hline
\end{tabular}


Continuação do Quadro 2

\begin{tabular}{|c|c|c|c|c|}
\hline $\begin{array}{l}\text { Primeiro } \\
\text { autor/ País/ } \\
\text { Ano }\end{array}$ & $\begin{array}{c}\text { Público-alvo } \\
\text { (População/amostra) } \\
\text { Desenho do estudo }\end{array}$ & $\begin{array}{c}\text { TIC } \\
\text { Recursos } \\
\text { Audiovisuais }\end{array}$ & Avaliação & Principais Resultados \\
\hline $\begin{array}{l}\text { Jonas } \\
\text { Reino } \\
\text { Unido } \\
2010\end{array}$ & $\begin{array}{c}\text { Estudantes de } \\
\text { enfermagem (18) } \\
\text { Transversal }\end{array}$ & $\begin{array}{c}\text { AVA } \\
\text { Links para } \\
\text { websites } \\
\text { úteis, leitura } \\
\text { complementar, } \\
\text { vídeos, fóruns } \\
\text { de discussão e } \\
\text { questões. }\end{array}$ & Avaliação do curso. & $\begin{array}{l}\text { Dor pediátrica } \\
\text { As avaliações foram positivas quanto aos módulos, } \\
\text { atividades, recursos, referências e navegabilidade. A } \\
\text { falta de tempo para estudo, pouca comunicação e } \\
\text { dificuldade de acesso à internet foram consideradas } \\
\text { desvantagens. }\end{array}$ \\
\hline $\begin{array}{l}\text { Bueno }^{(48)} \\
\text { Brasil } \\
2014\end{array}$ & $\begin{array}{c}\text { Profissionais e estudantes } \\
\text { da área da saúde (10) } \\
\text { Relato de Caso }\end{array}$ & $\begin{array}{l}\text { AVA } \\
\text { Exercícios, } \\
\text { imagens, vídeos, } \\
\text { textos e fóruns de } \\
\text { discussão. }\end{array}$ & $\begin{array}{c}\text { Pré-teste, pós-teste } \\
\text { e avaliação do } \\
\text { curso }\end{array}$ & $\begin{array}{l}\text { Dor neonatal } \\
\text { A média do pós-teste foi maior em relação ao } \\
\text { pré-teste. A carga horária, conteúdo e os recursos } \\
\text { foram positivamente avaliados. Uma das melhorias } \\
\text { sugeridas foi a inclusão de mais vídeos e imagens. }\end{array}$ \\
\hline
\end{tabular}

*Estudo que descreve dor neonatal e em adultos, optado por manter na tabela 2 por questões de formatação.

Algumas limitações também foram relatadas durante as avaliações de satisfação, como: falta de tempo dos usuários para finalização das atividades; falta de habilidade com as ferramentas de ensino; pouco contato dos alunos com seus pares; dificuldade de acesso à internet; dificuldades técnicas; e, mais especificamente, dificuldades na interpretação dos indicadores de dor dos pacientes virtuais.

Estudos apontaram, ainda, algumas sugestões mencionadas pelos usuários caracterizadas como melhorias técnicas e inclusão de recursos audiovisuais, a exemplo de estudos de casos clínicos, vídeos, animações e imagens.

\section{DISCUSSÃO}

Este estudo teve como objetivo identificar o uso de TICs empregadas no ensino da temática dor a estudantes e profissionais de saúde e o modo como as TICs são utilizadas. Uma ampla variedade de TICs e de recursos audiovisuais foram descritos nos estudos incluídos nesta revisão, em estudos de diferentes abordagens teóricas e metodológicas, o que permitiu uma descrição ampliada dos resultados.

A assistência adequada e eficaz na avaliação e controle da dor, é baseada em conhecimentos, competências e práticas dos profissionais e estudantes da área da saúde, pautados em evidências científicas ${ }^{(49)}$. A falta de conhecimento técnico-científico possibilita a influência de crenças e percepções pessoais na assistência, o que torna a educação na área de dor importante para o aprimoramento do conhecimento e habilidades dos profissionais e futuros profissionais da saúde(50).

Grande parte dos artigos incluídos nesta revisão destaca o impacto positivo da educação mediada por tecnologia no conhecimento dos estudantes e profissionais da saúde. Dos sete estudos que compararam o ensino mediado por tecnologia com o ensino presencial, seis apresentaram maior média de acertos dos participantes dos cursos online ${ }^{(28-32,38)}$ em relação aos alunos do ensino presencial. Além disso, observa-se resultados positivos na prática assistencial, exemplificadas pelo uso crescente de analgesia(24,42), redução da intensidade da dor de pacientes ${ }^{(42-20-21)}$ e aumento de registros de escores de dor em prontuários clínicos ${ }^{(44-24)}$. Os estudos mencionados acima e que demonstraram resultados positivos na prática clínica, são norte- americanos ${ }^{(42,44)}$ e australianos ${ }^{(21,24)}$, do tipo quasi-experimentaais ${ }^{(21,24,42,44)}$, sendo todos destinados à educação de enfermeiros e abordaram dor neonatal e pediátrica ${ }^{(42-44)} \mathrm{e}$ dor em indivíduos adultos ${ }^{(21,24)}$. 
Portanto, verifica-se a importância das TICs no ensino da dor, em diferentes categorias profissionais, e estudantes da área da saúde, não somente relacionadas ao aprendizado e satisfação dos alunos, mas também a respeito de mudanças na prática assistencial. Dessa forma, mais estudos que exploram a avaliação de estratégias de ensino em desfechos clínicos, como os relatados acima, são essenciais para a prática clínica.

As estratégias de educação mediadas por tecnologia constituem-se como alternativa ao ensino tradicional, visto que reúnem vantagens descritas nos estudos, tais como: flexibilidade temporal(15-16,18,41,48), desenvolvimento de autonomia e autoconfiança em relação ao aprendizado(31,45-48), aplicação dos conhecimentos apreendidos no AVA na prática clínica(24), estudo auto direcionado e centrado no usuário(27-46-18-48), desenvolvimento de competências e conhecimentos a partir de experiências de aprendizagem ativa ${ }^{(29,35,43)}$, maior custo benefício para o treinamento de profissionais ${ }^{(19-22,37,40)}$, ambiente motivador e engajador ${ }^{(20,45)}$, facilitação da comunicação entre os cursistas e professores/tutores ${ }^{(19)}$, ampliação de buscas rápidas por informações ${ }^{(19,48)}$ e atualização rápida e constante de novas evidências científicas ${ }^{(16-19,40)}$.

A implementação de TICs na educação demanda uma linguagem clara e objetiva, revisão das preferências e, principalmente, necessidades educacionais do público-alvo, além da utilização de recursos instrucionais interativos que incentivem o usuário(51). Os recursos audiovisuais podem possibilitar a aceitabilidade de uma estratégia educativa(52), como constatado nos estudos. As estratégias compostas por recursos como jogos, vídeos, imagens e simulados, obtiveram maior grau de avaliações positivas dos usuários ${ }^{(53)}$.

Entretanto, o uso de TICs também apresentam pontos negativos, relatados nesta revisão, como: falta de tempo dos usuários para finalização das atividades, e para concluírem o curso ${ }^{(19,21-22,29,40)}$, falta de habilidade com as ferramentas de ensino ${ }^{(17,40,47-19)}$, pouco contato dos alunos com seus pares ${ }^{(27,39-40)}$, dificuldade de acesso à internet ${ }^{(38-47)}$, dificuldades técnicas e de interpretação dos indicadores de dor dos pacientes virtuais ${ }^{(45)}$. Outras limitações podem ser atribuídas à educação mediada por TICs, a exemplo de: suporte técnico e gastos em hardware e software, ausência ou limitação em competências de informática por parte dos participantes, alta taxa de abandono dos $\operatorname{cursos}^{(53)}$, dificuldades em relação ao cadastro e ingresso em cursos on-line, perda de mensagens postadas, falta de familiaridade com o ambiente virtual e com a proposta do curso ${ }^{(54)}$.

Cabe destacar a lacuna do conhecimento na área das TICs no ensino da dor, no âmbito nacional, diante da identificação de somente três estudos nacionais ${ }^{(18-48-45)}$, abordando dor neonatal ${ }^{(48-45)}$ e em adultos ${ }^{(18-45)}$, corroborando para o desenvolvimento de pesquisas nacionais que englobem as TICs no ensino da dor com a finalidade de aumentar o conhecimento dos estudantes e profissionais da saúde, bem como apresentar mudanças na prática clínica.

\section{CONCLUSÃO}

Foram incluídos nesta revisão 34 estudos. Os AVA foram as TICs mais utilizadas para o ensino da dor a estudantes e profissionais da saúde, por meio de animações, exercícios interativos, gráficos, questões de múltipla escolha, textos, quizzes, imagens, vídeos, estudos de caso, auditoria, feedback, seção de ajuda aos usuários, áudios, links para websites, pacientes virtuais, tutoriais, elementos de videogames, exercícios, textos complementares, fórum de discussão, slides, palavras cruzadas, vídeo-aulas e podcasts.

No ensino, a utilização de TICs na educação, possibilita a construção do conhecimento pelo usuário, a partir da aprendizagem ativa, e não apenas a transmissão dos conteúdos, o que mostra ser um desafio para práticas pedagógicas atuais, proporcionando interatividade e dinamismo no processo de ensino. Dessa forma, salienta-se que, além de desenvolver novos recursos instrucionais com o emprego de TICs na área da saúde, avaliar aparência e conteúdo, e avaliar o processo de implementação, configura-se como primordial para a qualidade do ensino-aprendizado. 
Já para a área assistencial, o conhecimento dos profissionais e estudantes da área da saúde sobre a temática dor, advindos de TICs permite autonomia na tomada de decisões, considerando a facilidade que esta ferramenta proporciona, bem como a gestão pessoal no processo de aprendizagem com enfoque em mudanças na prática assistencial.

Para a pesquisa, verifica-se a necessidade de desenvolvimento de mais estudos nacionais que englobem as TICs no ensino da dor, resultando em posterior mudanças na assistência, beneficiando os usuários dos sistemas de saúde. Na gestão, a avaliação das necessidades da instituição, gerenciamento de pessoas e processo, e garantia de segurança ao paciente, são pontos a serem considerados para capacitação de pessoas em qualquer tema a ser abordado, considerando o uso de TICs e facilitar este processo de aprendizagem.

Ressalta-se, ainda, as limitações deste estudo que se relacionam ao reduzido número de estudos encontrados, às diferentes metodologias utilizadas, à diversidade de desfechos analisados e à amplitude do público-alvo que apesar de serem todos da área da saúde, possuem especificidades em suas profissões. A ampliação futura do estudo é possível considerando a inclusão de manuais, protocolos, relatórios de organizações e estudos de revisão.

\section{REFERÊNCIAS}

1. International Association for the Study of Pain. IASP pain terminology. 2008.

2. Carr ECJ, Briggs EV, Briggs M, Allcock N, Black P, Jones D. Understanding factors that facilitate the inclusion of pain education in undergraduate curricula: Perspectives from a UK survey. Br J Pain. 2016;10(2):100-7.

3. Alemán JLF, Carrillo de Gea JM, Mondéjar JJR. Effects of competitive computer-assisted learning versus conventional teaching methods on the acquisition and retention of knowledge in medical surgical nursing students. Nurse Educ Today. 2011;31:866-71.

4. Freire LM, Paula MA, Duarte ED, Bueno M. Distance education in neonatal nursing scenarios: a systematic review. Rev Esc Enferm USP. 2015;49(3):515-21.

5. Moore JL, Dickson-Deane C, Galyen K. e-Learning, online learning, and distance learning environments: Are they the same?. Internet High Educ. 2011;14:129-35.

6. Rodrigues RCV, Peres HHC. A panorama of Brazil's online nursing teaching. Rev Esc Enferm USP. 2008;42(2):298-304.

7. Prado C, Santiago LC, Silva JAM, Pereira IM, Leonello VM, Otrenti E, et al. Ambiente virtual de aprendizagem no ensino de Enfermagem: relato de experiência. Rev Bras Enferm. 2012;65(5):862-6.

8. Ruiz JG, Mintzer MJ, Leipzig RM. The impact of e-learning in medical education. Acad Med. 2006;81(3):207-12.

9. Cook DA, Garside S, Levinson AJ, Dupras DM, Montori VM. What do we mean by web-based learning? A systematic review of the variability of interventions. Med Educ. 2010;44:765-74.

10. Pereira ATC, Schmitt V, Dias MR, Álvares C. Ambientes virtuais de aprendizagem. In: Pereira, ATC, editor. Ambientes virtuais de aprendizagem: em diferentes contextos. Rio de Janeiro: Editora Ciência Moderna; 2007. p.2-22.

11. Arksey H, O'Malley L. Scoping studies: towards a methodological framework. Int J Social Res Methodol. 2005;8(1):19-32.

12. Levac D, Colquhoun H, O'Brien KK. Scoping studies: advancing the methodology. Implementation Science. 2010;5:69.

13. Moher D, Liberati A, Tetzlaff J, Altman DG, The PRISMA Group.Preferred Reporting Items for Systematic Reviews and Meta- Analyses: The PRISMA Statement. PLoS Med. 2009; 6(7):e1000097. 
14. Lizarondo L, Stern C, Carrier J, Godfrey C, Rieger K, Salmond S, et al. Mixed methods systematic reviews. In: Aromataris E, Munn Z, editors. Joanna Briggs Institute Reviewer's Manual. The Joanna Briggs Institute, 2017. Capitulo 8. Avaliable from: https://bit.ly/2m2N016

15. Huckstadt A, Hayes K. Evaluation of interactive online courses for advanced practice nurses. J Am Assoc Nurse Pract. 2005;17(3):85-89.

16. Dy SM, Hughes M, Weiss $C$, Sisson S. Evaluation of a web-based palliative care pain management module for housestaff. J Pain Symptom Manage. 2008;36(6):596-603.

17. Yanni LM, Priestley JW, Schlesinger JB, Ketchum JM, Johnson BA, Harrington SE. Development of a comprehensive e-learning resource in pain management. Pain Med. 2009;10(1):95-105.

18. Alvarez AG, Dal Sasso GTM. Aplicação de objeto virtual de aprendizagem, para avaliação simulada de dor aguda, em estudantes de enfermagem. Rev. Latino-Am Enfermagem. 2011;19(2):1-9.

19. Puljak L, Sapunar D. Web-based elective courses for medical students: an example in pain. Pain Med. 2011;12:854-63.

20. Borglin G, Bohman D. An online learning module improves specialist palliative care nurses' pain assessments and patient-reported pain. Evid Based Nurs. 2015;18(4):122.

21. Phillips JL,Heneka N, Hickman L, Lam L, Shaw T. Impact of a novel online learning module on specialist palliative care nurses' pain assessment competencies and patients' reports of pain: results from a quasi-experimental pilot study. J Palliat Med. 2014;28(6):521-29.

22. Slaughter AL, Frith K, O'Keefe L, Alexander S, Stoll R. Promoting best practices for managing acute low back pain in an occupational environment. Workplace Health Saf. 2015;63(9):408-14.

23. Friesgaard KD, Paltved C, Nikolajsen L. Acute pain in the emergency department: Effect of an educational intervention. Scandinavian Journal of Pain. 2017;15:8-13.

24. Phillips JL, Nicole Heneka, Hickman L. Lam L. Shaw T. Can A Complex Online Intervention Improve Cancer Nurses' Pain Screening and Assessment Practices? Results from a Multicenter, Pre-post Test Pilot Study. Pain Management Nursing. 2017;18(2):75-89.

25. Bonkowski SL, Gagne JC, Cade MB, Bulla SA. Evaluation of a Pain Management Education Program and Operational Guideline on Nursing Practice, Attitudes, and Pain Management. J Contin Educ Nurs. 2018;49(4):178-85.

26. Watt-Wattson J, McGillion M, Lax L, Oskarsson J, Hunter J, MacLennan C, et al. Evaluating an Innovative eLearning Pain Education Interprofessional Resource: A Pre-Post Study. Pain Med. 2018;20(1):37-49.

27. Johnson MI, Dewhurst D, Williams AD. Computer-based interactive tutorial versus traditional lecture for teaching introductory aspects of pain. Res Learning Tech. 1997;5(3):22-31.

28. Raffety B, Allendoerfer C, Minstrell J, Chabal C, Dunbar P, Nakamura Y. A Facet-based system for computer-assisted instruction in pain management for elderly patients. In: Anais do Simpósio Annual AMIA; 2000 out. 4-8; Los Angeles (Califórnia), USA. Los Angeles; 2000. p.670-74.

29. Harris Jr. JM, Elliott TE, Davis BE, Chabal C, Fulginiti JV, Fine PG. Educating generalist physicians about chronic pain: live experts and online education can provide durable benefits. Pain Med. 2008;9(5):555-63.

30. Brody AA, Groce-Wofford TM. Feasibility of implementing a web-based education program in geriatric pain and depression for home health care nurses. Home Health Care Manag Pract. 2013;20(10):1-5.

31. Weiner DK, Morone NE, Spallek H, Karp JF, Schneider M, Washburn C, et al. E-learning module on chronic low back pain in older adults: evidence of effect on medical student objective structured clinical examination performance. J Am Geriatr Soc. 2014;62(1):1161-67. 
32. Trudeau KJ, Hildebrand C, Garg P, Chiauzzi E, Zacharoff KL. A randomized controlled trial of the effects of online pain management education on primary care providers. Pain Med. 2017;18(4):680-69.

33. Jacobs ZG, Elnicki DM, Perera S, Weiner DK. An E-learning Module on Chronic Low Back Pain in Older Adults: Effect on Medical Resident Attitudes, Confidence, Knowledge, and Clinical Skills. Pain Med. 2018;19: 1112-20.

34. Sloan PA, LaFountain P, Plymale M, Johnson M, Snapp J, Sloan DA. Cancer pain education for medical students: the development of a short course on CD-ROM. Pain Med. 2002;3(1):66-72.

35. Douglass MA, Chan T, Gonyeau M, Lancaster JW, Woolley AB, DiVall M. Influences of experiential year and web-based learning module on student pharmacists' confidence and competence in pain management. Curr Pharm Teach Learn. 2015;7(1):94-99.

36. Fricton J, Anderson K, Clavel A, Fricton R, Hathaway K, Kang W, et al. Preventing chronic pain: a human systems approach - results from a massive open online course. Glob Adv Health Med. 2015;4(5):23-32.

37. Terpstraa JA, Van der Vaarta R, Spillekom-van Koulilb S, van Damc A, Rosmalend JGM, Knoope $\mathrm{H}$, van Middendorpa $\mathrm{H}$, Eversa AWM. Becoming an eCoach: training therapists in online cognitive-behavioral therapy for chronic pain. Patient Educ Couns. 2018;101:1702-7.

38. Keefe G, Wharrad HJ. Using e-learning to enhance nursing students' pain management education. Nurse Educ Today. 2012;32(1):66-72.

39. Jenkins MS, Bean WG, Luke K. Part-time, e-learning interprofessional pain management education for the primary and community care setting. Br J Pain. 2014;8(1):16-26.

40. Schmitt MB, Titler MG, Herr KA, Ardery G. Challenges of web-based education in educating nurses about evidence-based acute pain management practices for older adults. J Contin Educ Nurs. 2004;35(3):121-7.

41. Jansen BDW, Brazil K, Passmore P, Buchanan H, Maxwell D, McIlfatrick SJ, et al. Evaluation of the impact of telementoring using $\mathrm{ECHO}(\mathrm{C}$ technology on healthcare professionals' knowledge and self-efficacy in assessing and managing pain for people with advanced dementia nearing the end of life. BMC Health Serv Res. 2018;18(1):228.

42. Vincent $\mathrm{CVH}$, Wilkie DJ, Wang E. Pediatric nurses' beliefs and pain management practices: an intervention pilot. West J Nurs Res. 2011;33(6):825-45.

43. Ameringer S, Fisher D, Sreedhar S, Ketchum JM, Yanni L. Pediatric pain management education in medical students: impact of a web-based module. J Palliat Med. 2012;15(9):978-983.

44. Habich M, Letizia M. Pediatric pain assessment in the emergency department: a nursing evidence-based practice protocol. J Pediatr Nurs. 2015;41(4):198-202.

45. Alvarez AG, Dal Sasso GTM, Iyengar MS. Persuasive technology in teaching acute pain assessment in nursing: results in learning based on pre and post-testing. Nurse Educ Today. 2017;50:109-14.

46. Gibbins S, Maddalena P, Yamada J, Stevens B. Testing the satisfaction and feasibility of a computer-based teaching module in the neonatal intensive care unit. Adv Neonatal Care. 2007;7(1):43-9.

47. Jonas $D$, Burns $B$. The transition to blended e-learning: changing the focus of educational delivery in children's pain management. J Nurs Educ Pract. 2010;10(1):1-7.

48. Bueno M, Duarte ED, Marques RL, Freire LM, Castral TC. Neonatal pain assessment program II: an innovative strategy to increase knowledge translation-case report. Rev Dor. 2014;15(2):152-5. 
49. Rochman DL, Sheehan MJ, Kulich RJ. Evaluation of a pain curriculum for occupational therapists: experiences from a master's-level graduate program over six years. Disabil Rehabil. 2013;35(22):1933-40.

50. Zhang $\mathrm{CH}$, Hsu L, Zou BR, Li JF, Wang HY, Huang J. Effects of a pain education program on nurses' pain knowledge, attitudes and pain assessment practices in China. J Pain Symptom Manage. 2008;36(6):616-27.

51. Milic NM, Trajkovic GZ, Bukumiric ZM, Cirkovic A, Nikolic IM, Milin JS, et al. Improving Education in Medical Statistics: Implementing a Blended Learning Model in the Existing Curriculum. PLoS ONE. 2016;11(2):1-10.

52. Liu SH, Liao HL, Pratt JA. Impact of media richness and flow on e-learning technology acceptance. Comput Educ. 2009; 52:599-607.

53. Gallo AM. Beyond the Classroom: using technology to meet the educational needs of multigenerational perinatal nurses. J Perinat Neonat Nurs. 2011;25(2):195-9.

54. Veredas FJ, Ruiz-Bandera E, Villa-Estrada F, Rufino-González JF, Morente L. A web-based e-learning application for wound diagnosis and treatment. Comput Methods Programs Biomed. 2014;116:236-48. 